\title{
РАЗРАБОТКА ПРОГРАММЫ ПО ОЦЕНКЕ КАЧЕСТВА АКАДЕМИЧЕСКИХ ПРОГРАММНЫХ ПРОДУКТОВ НА ОСНОВЕ МЕТОДИКИ ХОАСТЕДА
}

\author{
(Работа выполнена при частичной поддержке гранта РФФИ, проект № 14-07-00665)
}

A.И. Афранасова, аспирант, младший научный сотрудник, nastyaafanasova@yandex.ru (Ульяновский государственный университет, ул. А. Толстого, 42, г. Ульяновск, 432000, Россия)

\begin{abstract}
Аннотация. В современном мире оценивание качества программного обеспечения считается одной из важнейших задач как в софтверных компаниях, так и в высших учебных заведениях с компьютерноориентированным уклоном подготовки. В таких вузах вопрос о том, как оценивать качество создаваемых студентами программ - так называемых академических программных продуктов, остается не вполне решенным, хотя его решение опосредованно затрагивает интересы и софтверных компаний, куда придут работать выпускники. Ввиду существования ряда отличительных особенностей академических программных продуктов от профессиональных оценивание их качества требует специального подхода и особой вычислительной методики.

В статье излагаются принципиальные особенности методики оценивания качества академических про-граммных продуктов, разработанной и применяемой в Ульяновском государственном университете для оце-нивания качества программ, написанных студентами на $\mathrm{C}++$. Описана оригинальная, созданная на языке С\# «Программа по оценке качества академических программных продуктов на основе методики Холстеда» для автоматизации процесса оценивания.
\end{abstract}

Ключевые слова: оценивание качества программных продуктов, академические программные продукты, программометрия, метрики Холстеда, проектно-ориентированное обучение, $C++$.

Оценивание качества ПО считается в настоящее время одной из важнейших задач, стоящих и перед софтверными компаниями, и перед высшими учебными заведениями с компьютерно-ориентированным уклоном подготовки. Вопрос о том, как оценивать качество создаваемых студентами программ - так называемых академических программных продуктов (АПП), в таких вузах не вполне решен, хотя его решение опосредованно затрагивает интересы и софтверных компаний, куда придут работать выпускники. Так как между АПП и профессиональными программными продуктами (ППП) существует ряд отличий, оценивание их качества требует специального подхода и особой вычислительной методики.

Задаче оценивания качества программных продуктов посвящено множество исследований [1-5], однако их объектом, как правило, являются ППП. Задаче оценивания качества АПП уделяется значительно меньшее внимание, хотя эта задача неизбежно возникает на технических специальностях, где студенты в ходе учебного процесса создают большое количество программ [5-8]. В силу особенностей АПП для обработки таких больших массивов проектной документации требуются специальные средства.

В отличие от ППП в АПП отсутствует обратная связь по их качеству, поскольку АПП в большинстве случаев не имеют непосредственного контакта с конкурентной рыночной средой. Оценивание качества студенческих программ обычно выполняется экспертами - преподавателями, которые, формируя оценку, опираются на свой личный опыт, знания и представления. Однако даже при условии высокой квалификации преподавателей их требования индивидуальны, субъективны и существуют на интуитивном уровне. Учитывая то, что во время обучения создается большое количество АПП, их оценивание - процесс достаточно сложный, затратный по времени и трудоемкий. Сведений о средствах автоматизации именно этого процесса в литературе явно недостаточно.

Данная работа предпринята с целью восполнения отмеченного недостатка. Ее выполнение в Ульяновском государственном университете обусловлено тем, что здесь при чтении дисциплин, связанных с вычислительной математикой и программированием, с 1989 года применяется проекто-ориентированное обучение [8, 9], которое предусматривает разработку студентами целостных, нетривиальных программных продуктов. Оценив уровень качества по множеству таких АПП, преподаватель и кафедра получают необходимую обратную связь и, следовательно, возможность формирования оценки качества всего образовательного процесса [10].

\section{Процедуры оценивания качества программных продуктов, классификация существующих методов}

Одной из важнейших проблем оценивания качества программных продуктов является формализация понятия оценки качества, характеристик качества, а также методология их оценивания. 

ты.

Оцениванию качества программных средств посвящены государственные и международные стандар-

В ГОСТ 28195-89 дается определение процедуры оценки качества программного средства как совокупности операций, включающей выбор номенклатуры показателей качества, определение значений этих показателей и сравнение их с базовыми значениями.

Существующие подходы к оцениванию качества программных продуктов можно классифицировать следующим образом [11].

- По внутренним показателям качества. К ним относят подходы, основанные на оценке ряда технологических показателей программы (таких, как сложность программы, вычислительная неизбыточность, структурная целесообразность и т.д.). Внутренние показатели качества программного продукта оказывают влияние на его внешние показатели.

- По внешним показателям качества. К оценке качества по внешним показателям относят подходы, основанные на оценке различного рода показателей, непосредственно наблюдаемых при испытаниях программных средств.

- Смешанный подход. К смешанным относят подходы, основанные на оценивании значений как внешних, так и внутренних показателей качества.

При разработке методологии оценивания качества АПП важно понимать, что на этапе обучения студентам в первую очередь необходимо научиться писать код, обладающий качественными внутренними характеристиками. А учитывая тот факт, что у АПП, как правило, отсутствует обратная связь от пользователя, целесообразным является оценивание АПП именно по их внутренним показателям качества.

\section{Методика оценивания качества АПП}

В основу предлагаемой методики положим работу [12], применение которой для оценивания качества АПП впервые предложено в [10].

В соответствии с методикой Холстеда оценку качества программного продукта формируют в результате определения специальных характеристик (таких, как число операторов и операндов) исходного кода программы. При этом априори считается, что текст программы свободен от ошибок, связанных с языком программирования. Для АПП это справедливо, поскольку для оценивания отбираются тексты программ, успешно прошедшие компиляцию. Также считают, что текст АПП свободен от логических (алгоритмических) ошибок и отклонений от выданного задания, поскольку он уже прошел необходимую проверку у эксперта-преподавателя.

Процесс получения качественных характеристик АПП. Охарактеризуем процесс получения параметров качества АПП.

По методике Холстеда [12], принципиальным параметром качества АПП является критерий $H q$ совершенства логической структуры текста программного продукта. Критерий $H q$ вычисляют с использованием четырех характеристик длины программного текста:

$\eta_{1}$ - число простых (или отдельных) операторов, появляющихся в данной реализации (словарь операторов); дов);

$\eta_{2}$ - число простых (или отдельных) операндов, появляющихся в данной реализации (словарь операн-

$\mathrm{N}_{1}$ - общее число всех операторов, появляющихся в данной реализации;

$\mathrm{N}_{2}$ - общее число всех операндов, появляющихся в данной реализации.

На основе этих параметров для каждого текста АПП вычисляют две характеристики длины: экспериментально определенную

$$
\mathrm{N}=\mathrm{N}_{1}+\mathrm{N}_{2}
$$

и теоретическую

$\hat{\mathrm{N}}=\eta_{1} * \log _{2} \eta_{1}+\eta_{2} * \log _{2} \eta_{2}$.

Обоснование корректности формулы вычисления теоретической длины. По данным работы [10], формула (2) имеет строгое математическое доказательство в [13]. Она выражает теоретическую длину $\hat{\mathrm{N}}$ программы через число входящих в ее код простых операторов $\eta_{1}$ и число простых операндов $\eta_{2}$, исходя из минимаксного для данного типа программ так называемого «стоимостного» критерия. Это доказательство основано на предположении, что при создании программы реализуется функциональная задача в максимально экономной и рациональной форме. В [11] введено соотношение

$$
\mathrm{N}=\lambda\left(\eta_{1} * \log _{2} \eta_{1}+\eta_{2} * \log _{2} \eta_{2}\right)=\lambda \hat{\mathrm{N}}
$$

где $\lambda$ - параметр, характеризующий степень приближения N к $\hat{\mathrm{N}}$. Там же выполнено экспериментальное обоснование, в ходе которого (после обработки десяти профессионально составленных программ на языках высокого уровня) получено значение $\lambda=0,934336314$, достаточно близкое к 1. 
В литературе по методам программометрии используются и другие методы измерения характеристик программ. Тем не менее, программы, для которых значение $\lambda$ приближается к 1, общепринято классифицировать как близкие по качеству к профессиональным программам, что и обосновывает выбор метрики Холстеда в данной работе.

Статистическая обработка программных текстов. После того как все тексты АПП обработаны и для каждого из них уже вычислены соответствующие характеристики - экспериментальная и теоретическая длины, осуществляем обработку полученных параметров методами математической статистики.

Для выборки теоретических значений длин программ и выборки экспериментально вычисленных значений длин программ, где объем каждой выборки (количество обрабатываемых файлов) равен $n$, находим расчетные значения оценок статистических характеристик:

1. Математическое ожидание:

$\tilde{M}_{x}=\frac{1}{n} \sum_{i=1}^{n} x_{i}$

2. Дисперсия:

$\tilde{D}_{x}=\frac{\sum_{i=1}^{n}\left(x_{i}-\tilde{M}_{x}\right)^{2}}{n-1}$.

3. Среднеквадратичное отклонение:

$\tilde{\sigma}_{x}=\sqrt{\tilde{D}_{x}}$.

Далее, когда оценка статистических характеристик выполнена, для теоретически полученной и экспериментально определенной длин АПП вычисляем коэффициент корреляции:

$$
\tilde{r}_{x y}=\frac{\sum_{i=1}^{n}\left(x_{i}-\tilde{M}_{x}\right) \cdot\left(y_{i}-\tilde{M}_{y}\right)}{(n-1) \cdot \tilde{\sigma}_{x} \cdot \tilde{\sigma}_{y}} .
$$

Критерий совершенства $H q$ численно равен коэффициенту корреляции $\tilde{r}_{x y}$.

Для совершенных программ параметр $H q$ близок к 1 , для программ, имеющих тот или иной набор несовершенств, отличия теоретического и экспериментально определенного значений длины текста будут более значительны, поэтому в результате коэффициент корреляции $\tilde{r}_{x y}$ уменьшится, то есть уменьшится параметр $\mathrm{Hq}$.

Поскольку на начальном этапе создания программ особое внимание уделяется правильному, оптимальному построению алгоритма и реализации его на языке программирования, параметр $H q$ является важным для оценки качества академических программных продуктов.

Программа оценивания качественных характеристик АПП по методике Холстеда. Охарактеризуем программную реализацию вычислений, описанных выше.

Разработанная программа [14] представляет собой специализированную программу для автоматического определения качественных характеристик академических программных продуктов на языке $\mathrm{C}++$ на основе изложенной методики.

Программа реализована в среде Visual Studio на языке программирования С\# и состоит из основной функции, функции лексического анализатора Analyzer() и функции синтаксического анализатора Result().

Процесс обработки программного текста организован следующим образом.

1. Основная функция программы считывает посимвольно текст каждого АПП из указанного пользователем подкаталога и отправляет каждый символ в лексический анализатор. Блок-схема алгоритма работы основной функции программы представлена на рисунке 1.

2. Лексический анализатор на основе поступающих на вход символов последовательно формирует лексему. Когда лексема сформирована, она передается в синтаксический анализатор. Схема конечного автомата лексического анализатора представлена на рисунке 2.

3. Синтаксический анализатор сопоставляет входную лексему с грамматикой языка, которая описана в программном коде в виде статических строковых массивов, и определяет, к какому типу относится входная лексема. В функции учитывается возможность ситуации, когда две различные переменные, находящиеся в разных областях видимости - локальная и глобальная, имеют одинаковое имя. Такие переменные фиксируются анализатором как два разных операнда. После того как определено, к какому классу относится поступившая на вход лексема, изменяется общее количество операторов или операндов программы, а также при необходимости пополняются словари операторов и операндов программы. 


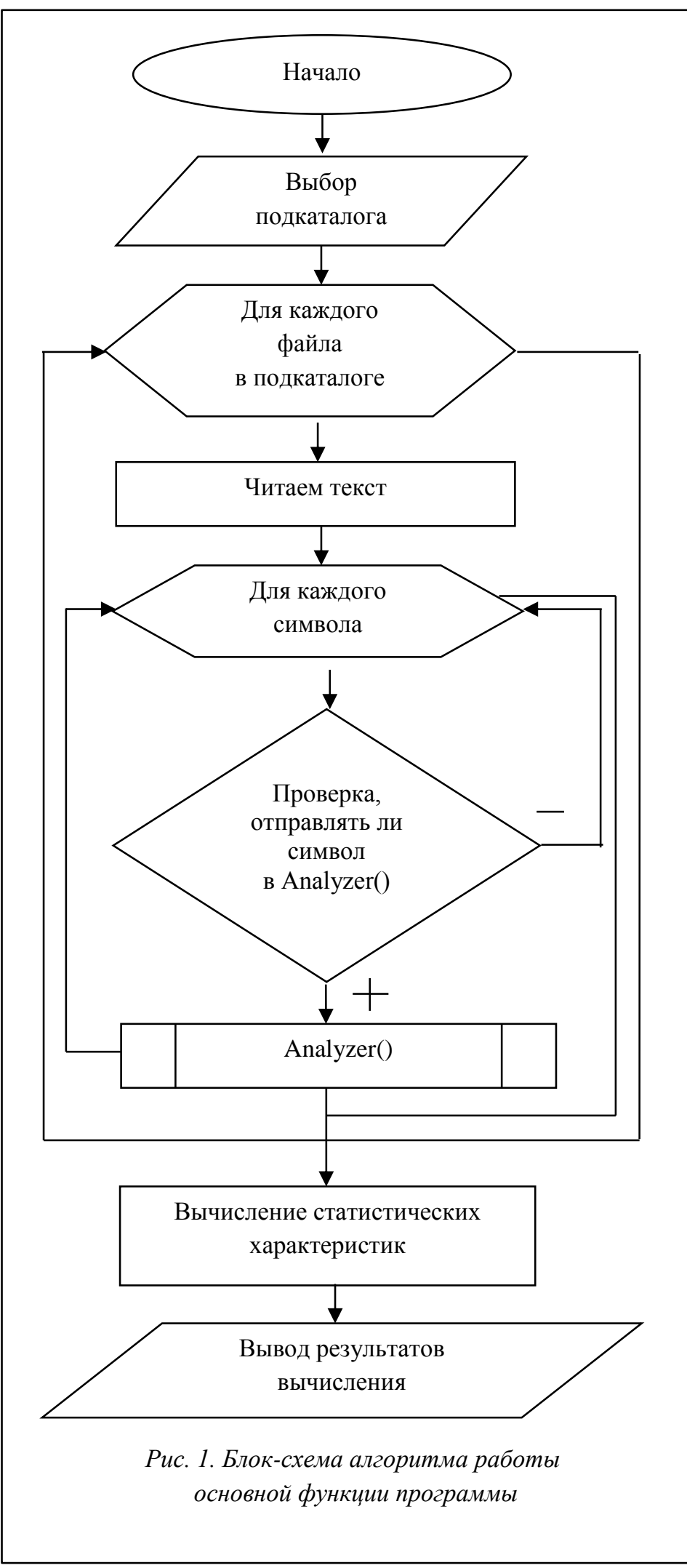

4. Далее, когда текст программы полностью обработан, в основной функции программы вычисляется его экспериментальная и теоретическая длина.

После окончания обработки всех программных текстов АПП для всей группы текстов выполняется вычисление оценок статистических характеристик (формулы (4)-(6)).

Далее на основе полученных данных по формуле (7) вычисляется критерий совершенства Hq. Результаты работы программы выводятся на экран.

Опишем взаимодействие программы и пользователя.

Для запуска процесса получения качественных характеристик пользователю необходимо выбрать подкаталог файлов - текстов АПП для их дальнейшей обработки. После того как пользователем указан необходимый подкаталог файлов, для каждого программного текста происходит формирование и вычисление следующих параметров качества: словарь операторов $\eta_{1}$, словарь операндов $\eta_{2}$, общее число всех операторов $\mathrm{N}_{1}$, общее число всех операндов $\mathrm{N}_{2}$, экспериментально полученная длина $\mathrm{N}$ и теоретическая вычисленная длина $\hat{\mathrm{N}}$ параметры выводятся на экран в виде таблицы. Под таблицей выводится вычисленное значение параметра совершенства для всей группы файлов. Наглядно это показано на рисунке 3 , где представлены результаты обработки АПП студентов специальности «Информационные системы» факультета математики и информационных технологий УлГУ, выполненных в 2013-2014 учебном году в рамках дисциплины «Технология программирования» по теме «Вычисления по формулам».

В таблице представлены экспериментальные данные, полученные с помощью разработанной программы при оценивании лабораторных работ по дисциплине «Технология программирования» для студентов специальности «Информационные системы» факультета математики и информационных технологий УлГУ, выполненных также в 2013-2014 году. По количеству выполненных работ и параметру совершенства можно судить об уровне освоения изученной темы. Например, в рамках тем 3-5 сдано мало работ, однако параметр качества для них высок. Из этого следует, что тему освоило небольшое количество студентов, но, по-видимому, это отличники. Количество работ по теме 6 тоже невелико, а параметр совершенства значительно ниже, чем по всем предыдущим темам. Здесь можно сделать вывод о сложности данной темы и необходимости усовершенствовать преподнесение материала в рамках семинарских занятий. 

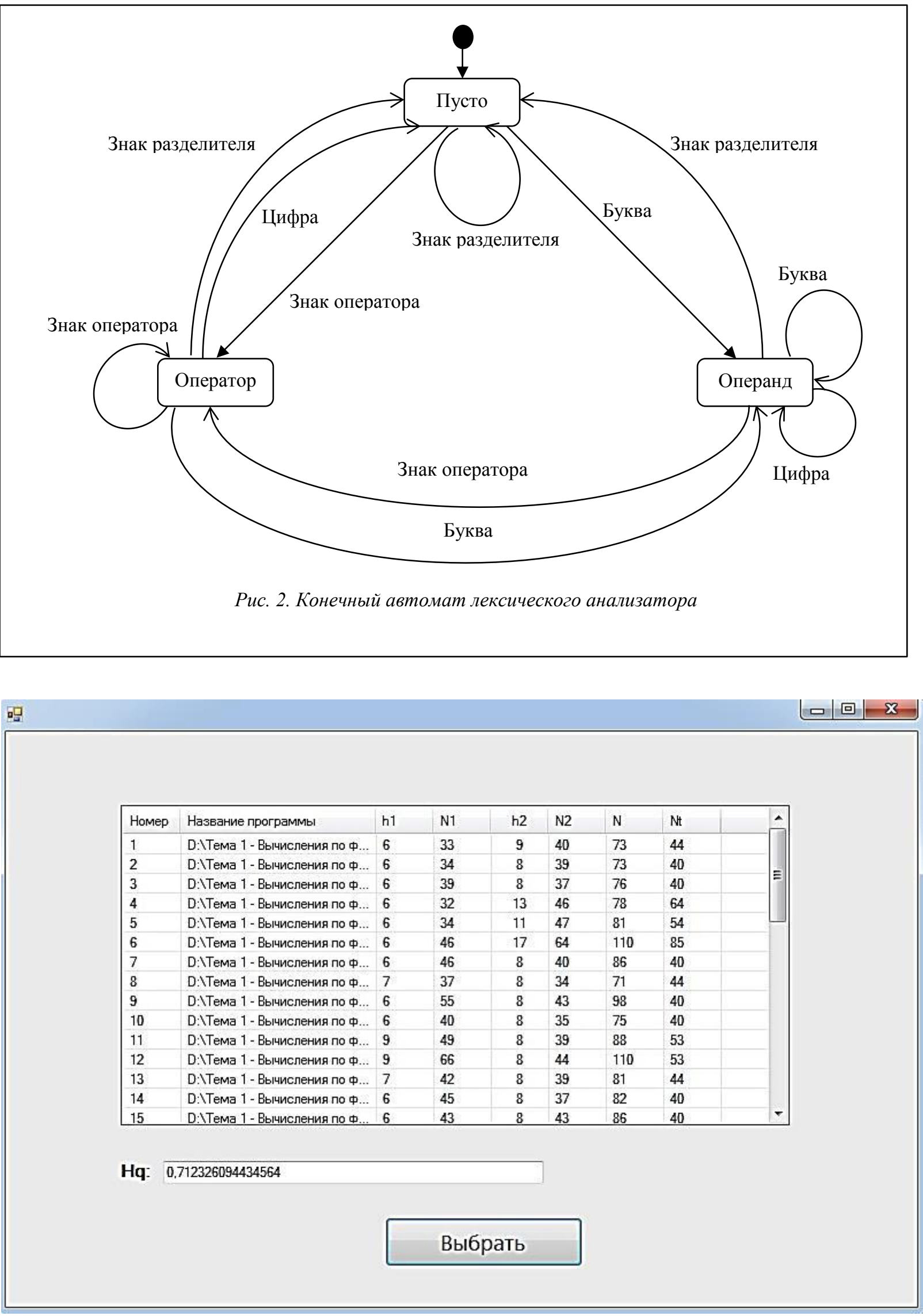

Рис. 3. Пример результата работы программы 
Экспериментальные результаты определения параметра совершенства

\begin{tabular}{|c|l|c|l|}
\hline \multicolumn{1}{|c|}{ Тема } & Количество работ & Параметр совершенства Нq \\
\hline 1 & Вычисления по формулам & 40 & 0,712326094434564 \\
\hline 2 & Операторы цикла & 23 & 0,567967833381929 \\
\hline 3 & Одномерные массивы & 8 & 0,851011658832649 \\
\hline 4 & Последовательности & 3 & 0,96756114421808 \\
\hline 5 & Обработка текстовых строк & 3 & 0,825354659308643 \\
\hline 6 & Матрицы & 4 & 0,681555060203852 \\
\hline
\end{tabular}

Таким образом, объективные и проверяемые индикаторы качества АПП являются мощным инструментом модификации поведения студентов и преподавателей. Реализованные в специальных программах тестирования АПП обладают рядом достоинств по сравнению с традиционными практиками: исключают риск манипулирования, обеспечивают многоаспектное понимание результатов деятельности кафедры, отдельного преподавателя и группы (потока) студентов, изучающих дисциплину, допускают прямое сравнение различных методик преподавания и являются публично доступными и легко интегрируемыми в учебный процесс. Применение таких индикаторов находит положительные отзывы как со стороны преподавателей (снижает трудоемкость аттестации студентов, автоматизируя этот процесс), так и студентов (стимулирует стремление к достижению лучших показателей).

Созданная «Программа оценки качественных характеристик академических программных продуктов на основе методики Холстеда» зарегистрирована в Роспатенте [14] и прошла успешную опытную эксплуатацию на материале лабораторных проектов дисциплины «Технология программирования», в рамках которой было обработано 86 АПП, созданных в 2013-2014 гг.

Планируется продолжение опытной эксплуатации разработанной программы на расширенном наборе дисциплин, находящихся в учебном процессе факультета математики и информационных технологий УлГУ, а именно: «Технология программирования», «Языки программирования и методы трансляции», «Методы программирования», «Объектно-ориентированное программирование», «Численные методы», «Методы вычислений», «Вычислительная математика» и других, где учебным планом предусмотрены лабораторные работы в форме академических программных продуктов. Для этого создается банк АПП. В настоящее время он содержит 151 запись.

\section{Лuтература}

1. Interface.ru. Interface and software company. URL: http://www.interface.ru/home.asp?artId=3987 (дата обращения: 18.10.2015).

2. Антошина И.В., Домрачев В.Г., Ретинская И.В. Основные тенденции оценивания качества программных средств // Качество, инновации, образование. 2004. № 1. С. 70-75.

3. Калинина Л.Ю. Оценка качества программных продуктов // Качество, инновации, образование. 2006. № 4. С. 52-55.

4. Антошина И.В., Домрачев В.Г., Ретинская И.В. Методика составления системы характеристик качества для программных средств // Качество, инновации, образование. 2002. № 3. С. 57-60.

5. Липаев В.В. О проблемах оценивания качества программных средств // Качество, инновации, образование. 2002. № 1. С. 93-97.

6. Информационно-коммуникационные технологии в оRL: http://www.ict.edu.ru/vconf/index.php? $\mathrm{a}=\mathrm{v} \operatorname{conf} \& \mathrm{c}=$ getForm \&r=thesisDesc\&d=light\&id_sec=123\&id_thesis=46 43 (дата обращения: 12.11.2015).

7. Информационные технологии в образовании: конгресс конференций. URL: http://ito.edu.ru/2003/VI/VI-0-1548.html (дата обращения: 12.11.2014).

8. Семушин И.В., Угаров В.В. Модификация поведения студента и преподавателя инженерных дисциплин // Московское научное обозрение. 2013. № 9 (37). С. 3-8.

9. Семушин И.В., Угаров В.В., Цыганова Ю.В., Афанасова А.И., Куличенко И.Н. Опыт проектноориентированного обучения в университетах Ульяновска // Перспективные информационные технологии (ПИТ 2014): тр. Междунар. науч.-технич. конф.; [под ред. С.А. Прохорова]. Самара: Изд-во Самарского науч. центра РАН, 2014. С. 436-438.

10. Угаров В.В. Компьютерные модели и программные комплексы в проектно-ориентированном обучении: дис. ... канд. технич. наук. Ульяновск, 2005.

11. Антошина И.В., Домрачев В.Г., Ретинская И.В. Основные тенденции оценивания качества программных средств // Качество, инновации, образование. 2004. № 1. С. 70-75.

12. Холстед М.Х. Начала науки о программах; [пер. с англ. В.М. Юфы]. М.: Финансы и статистика, $1981.128 \mathrm{c}$ 
13. Апостолова Н.А., Гольдштейн Б.С., Зайдман Р.А. О программометрическом подходе к оценкам программного обеспечения // Программирование. 1995. № 4. С. 38-44.

14. Афанасова А.И. Программа оценки качественных характеристик академических программных продуктов на основе методики Холстеда. Пат. 2015611297 РФ № 2014662403; заявл. 03.12.2014; опубл. 27.01.2015. 